\title{
MAP Kinases are Required for Apoptosis of HCT-8 Epithelial Cells Induced by the Parasite Giardia intestinalis
}

Pasqua Cavallo ${ }^{1}$, Antonia Cianciulli ${ }^{1}$, Rosa Calvello² ${ }^{2}$ Teresa Dragone ${ }^{1}$ and Maria Antonietta Panaro ${ }^{1 *}$

${ }^{1}$ Department of Scienze Mediche di Base, University of Bari, P.zza G. Cesare, 11, I-70124 Bari, Italy

${ }^{2}$ Department Farmaco-Biologico, University of Bari, University of Bari, P.zza G. Cesare, 11, I-70124 Bari, Italy

\begin{abstract}
Giardia intestinalis is a protozoan that causes a generally self-limited clinical illness typically characterized by diarrhea, abdominal cramps, bloating, weight loss and malabsorption. The pathogenesis of giardiasis is multifactorial and probably different in various animal models, but the mechanisms responsible for the disease are still poorly understood. We previously reported that $G$. intestinalis is able to induce apoptosis in the human HCT-8 epithelial cell line through the activation of both the intrinsic and extrinsic apoptotic pathways. In the present study we demonstrate that activation of the mitogen-activated protein kinases (MAPKs) plays an important role in the regulation of HCT-8 cell apoptosis induced by $G$. intestinalis. MAPK activation seems to correlate with regulation of the apoptotic process because specific MAPK inhibitors significantly reduced the expression of the active form of caspase-3 in infected cells. Apoptotic changes were also dramatically inhibited by pre-treatment of the cells with JNK or p38 specific inhibitors, but not ERK $1 / 2$ inhibitor. Taken together, these results suggest a critical role for MAPK activation in $G$. intestinalis-induced apoptosis in the human HCT-8 cell line.
\end{abstract}

Keywords: MAPK; Apoptosis; Giardia; HCT-8; Protozoa

\section{Introduction}

Giardia intestinalis (also known as G. lamblia and G. duodenalis) is a deeply divergent protist that causes intestinal infection worldwide. Infection can cause a generally self-limited clinical illness (i.e. giardiasis) typically characterized by diarrhea, abdominal cramps, bloating, weight loss and malabsorption, though asymptomatic infection frequently occurs [1-3]. Several reports have associated giardiasis with the development of chronic enteric disorders, allergies and reactive arthritis [4-7].

The parasite exists in two forms: infectious cysts and diseasecausing trophozoites. In the gut lumen, trophozoites differentiate into cysts, which are passed in the host's feces. Cysts have a filamentous wall that is resistant to harsh environmental conditions, facilitating the spread of giardiasis. For this reason, G. intestinalis poses a considerable problem, especially in the developing world where hygiene conditions are inadequate for blocking its transmission by the fecal-oral route.

Giardia species are ubiquitously distributed and have been detected in nearly all classes of vertebrates, including domestic animals and wildlife [8]. Recent advances in molecular epidemiology have led to the identification of specialized genetic groups (i.e., assemblages) that are relatively species-specific. Assemblages A and B of G. intestinalis primarily infect humans and primates, assemblages $\mathrm{C}$ and $\mathrm{D}$ infect dogs, assemblage F infects cats, assemblage E infects hoofstock and assemblage $\mathrm{G}$ infects rodents [9]. Although G. intestinalis infects both humans and animals, the role of zoonotic transmission to humans and the importance of animal contamination of food and water are being re-examined.

The pathogenesis of giardiasis is multifactorial and the mechanisms responsible for the disease are poorly understood [10]. We previously reported that $G$. intestinalis is able to induce apoptosis in the human HCT-8 epithelial cell line. In particular, we observed activation of both the intrinsic and extrinsic apoptotic pathways, down-regulation of the anti-apoptotic protein $\mathrm{Bcl}-2$ and up-regulation of the pro-apoptotic Bax, suggesting a possible role of caspase-dependent apoptosis in the pathogenesis of giardiasis [11]
The mitogen-activated protein kinases (MAPKs), a family of related serine/threonine protein kinases, constitute a large kinase network that regulates a variety of physiological processes, such as cell growth, differentiation and apoptotic cell death [12].

The MAPKs family, comprising the extracellular signal regulated kinases (ERKs), c-Jun $\mathrm{NH}_{2}$-terminal kinase (JNK) and p38 MAPK, is of particular interest because its activation is involved in cell survival [13]. After MAPKs (ERK1, 2, JNK1-3 and p38) are activated either in the cytoplasm or in the nucleus, they bind and regulate transcription by modulating the function of a target transcription factor through serine/ threonine phosphorylation [14]. In addition to the transcriptional effects of MAPKs signaling, accumulating evidence indicates that MAPKs regulate cell behavior also by phosphorylating cytoplasmic target proteins, such as apoptotic proteins [12].

In order to decipher the mechanisms underlying programmed cell death (PCD) triggered by G. intestinalis infection of the intestinal cell, the molecules involved in cellular signaling were investigated. In particular, we investigated the role of MAPKs activation in the apoptosis of Giardia-infected cells.

\section{Materials and Methods}

\section{Parasites}

G. intestinalis strain WB clone 6 was used for this study.

*Corresponding author: Maria Antonietta Panaro, Department of Scienze Mediche di Base, University of Bari, P.zza G. Cesare, 11, I-70124 Bari, Italy. Phone: (+39) 080 5478351; Fax: (+39) 080 5478325; E-mail: ma.panaro@anatomia.uniba.it

Received December 09, 2011; Accepted March 02, 2012; Published March 06 2012

Citation: Cavallo P, Cianciulli A, Calvello R, Dragone T, Panaro MA (2012) MAP Kinases are Required for Apoptosis of HCT-8 Epithelial Cells Induced by the Parasite Giardia intestinalis. J Microbial Biochem Technol 4: 031-036. doi:10.4172/1948-5948.1000068

Copyright: $\odot 2012$ Cavallo $P$, et al. This is an open-access article distributed unde the terms of the Creative Commons Attribution License, which permits unrestricted use, distribution, and reproduction in any medium, provided the original author and source are credited 
Trophozoites were grown axenically at $37^{\circ} \mathrm{C}$ in Keister's modified TYI-S-33 medium [15], ( $\mathrm{pH} 7.0)$ supplemented with bovine bile $(0.52$ $\mathrm{mg} / \mathrm{ml}$, Difco) and $10 \%$ heat-inactivated fetal bovine serum (FBS), $100 \mathrm{U} / \mathrm{ml}$ penicillin, $100 \mathrm{mg} / \mathrm{ml}$ streptomycin, all from Sigma-Aldrich (Milan, Italy).

\section{Cell cultures}

Cells of the human ileocecal adenocarcinoma line HCT-8 (ATCC CCL-244) were cultured, in controlled atmosphere $\left(5 \% \mathrm{CO}_{2}\right.$ atmosphere at $37^{\circ} \mathrm{C}$ ) in RPMI 1640 medium (Sigma) supplemented with sodium pyruvate $(1 \mathrm{mM}), 10 \%$ heat-inactivated FBS, $100 \mathrm{U} / \mathrm{ml}$ penicillin, $100 \mathrm{mg} / \mathrm{ml}$ streptomycin and L-glutamine $(2 \mathrm{mM})$. Since duodenal and colonic epithelial cells are physiologically similar, cocultivation of Giardia trophozoites with colonic epithelial cells was chosen in order to investigate parasite-host cell interactions.

The mycoplasma infection status was routinely investigated in our cell cultures using a PCR Mycoplasma Detection Set (Takara, \# 6601, Lonza, Milan, Italy) according to manufacturer's instruction. Negative controls were included in every PCR experiment to ensure the absence of contamination.

\section{Infection of cells with $G$. intestinalis trophozoites}

Trophozoites were harvested by cold shock on ice for $30 \mathrm{~min}$ and centrifuged at $350 \mathrm{~g}$ for $10 \mathrm{~min}$ at $4^{\circ} \mathrm{C}$. Then, after washes, live trophozoites were counted with a hemocytometer, added to cell cultures at a $3: 1$ (parasite/cell) ratio and incubated at $37^{\circ} \mathrm{C}$ in a $5 \% \mathrm{CO}_{2}$ atmosphere for different times (10 min-24 h) in order to establish the optimal time for observing MAPKs activation and cell apoptosis.

\section{Evaluation of apoptotic cells}

Apoptosis was morphologically assessed using the Vibrant Apoptosis YO-PRO assay (Molecular Probes, Eugene, OR, USA) according to the manufacturer's instructions in cell cultures subjected to infection with $G$. intestinalis trophozoites or treatment with the pro-apoptotic agent actinomycin D (5 mg/ml; Sigma). Untreated and uninfected cells were used as controls. Treated and untreated cells were incubated for $10 \mathrm{~min}$ to $24 \mathrm{~h}$ at $37^{\circ} \mathrm{C}$ in a $5 \% \mathrm{CO}_{2}$ atmosphere. Green fluorescent lipid dye YO-PRO-1 (fluorescein isothiocyanate), which can enter apoptotic cells and red fluorescent propidium iodide (PI; tetramethyl rhodamine isothiocyanate), which can only enter necrotic cells, were added to the cell culture for $30 \mathrm{~min}$ on ice. The percentages of cells exhibiting apoptosis and necrosis were then calculated using a fluorescence microscope with a 10x objective by counting at least 300 cells. Results were expressed as mean \pm SD of five different experiments.

\section{SDS-PAGE and Western blotting}

We analyzed the activation of MAPK subtypes ERK1/2, p38 and JNK by submitting cell lysates to sodium dodecyl sulfate polyacrylamide gel electrophoresis (SDS-PAGE) and Western blotting. Cells were incubated with or without $G$. intestinalis trophozoites. Intestinal cells pre-treated with or without various pharmacological inhibitors were incubated in the absence or presence of viable trophozoites. Briefly, after treatment, cells were washed twice in PBS, detached with ice-cold PBS, collected and centrifuged at $600 \mathrm{~g}$ for $10 \mathrm{~min}$. The supernatant was then removed and the pellet incubated with lysis buffer $[1 \%(\mathrm{v} / \mathrm{v})$ Triton X- 100, 20 mM Tris-HCl, $137 \mathrm{mM} \mathrm{NaCl,} \mathrm{10 \%} \mathrm{(v/v)} \mathrm{glycerol,}$ $2 \mathrm{mM}$ EDTA, $1 \mathrm{mM}$ phenylmethylsulfonyl fluoride (PMSF), $20 \mathrm{mM}$ leupeptin hemisulfate salt, $0.2 \mathrm{U} / \mathrm{ml}$ aprotinin (all from Sigma)] for $30 \mathrm{~min}$ on ice and then vortexed and centrifuged at $12,800 \mathrm{~g}$ for 10 min. The protein concentration in the supernatant was determined by Bradford's protein assay [16] and the lysate subjected to SDS-PAGE. Protein samples were diluted with sample buffer $[0.5 \mathrm{M}$ Tris- $\mathrm{HCl}, \mathrm{pH}$ 6.8, $10 \%(\mathrm{v} / \mathrm{v})$ glycerol, $10 \%(\mathrm{w} / \mathrm{v})$ SDS, $5 \%(\mathrm{v} / \mathrm{v}) \beta$-mercaptoethanol, $0.05 \%(\mathrm{w} / \mathrm{v})$ bromophenol blue] and then boiled for $3 \mathrm{~min}$. Protein (25 mg) and pre-stained standards (BioRad Laboratories, Hercules, CA, USA) were loaded on pre-cast $15 \%$ (for all proteins) SDS polyacrylamide gels (BioRad Laboratories).

After electrophoresis, the resolved proteins were transferred to nitrocellulose membranes. A blotting buffer [20 mM Tris/150 $\mathrm{mM}$ glycine, $\mathrm{pH} 8$ and $20 \%(\mathrm{v} / \mathrm{v})$ methanol] was used for gel and membrane saturation and blotting. The blotting conditions were $200 \mathrm{~mA}$ (constant) and $200 \mathrm{~V}$ for $110 \mathrm{~min}$. Blots were then blocked with PBS ( $\mathrm{pH} 7.2)$ containing $0.1 \%(\mathrm{v} / \mathrm{v})$ Tween 20 and 5\% (w/v) nonfat dried milk for $1 \mathrm{~h}$ and washed three times with $0.1 \%$ Tween 20-PBS (T-PBS). Membranes were then incubated for $60 \mathrm{~min}$ with the following antibodies (Santa Cruz Biotechnology, Santa Cruz, CA, USA): rabbit anti- $\beta$-actin pAb (sc-10 731, 1:200); and Abs against non-phosphorylated or phosphorylated forms of ERK1/2 [sc-153 (rabbit), 1:100 and sc-7976 (goat), 1:100 respectively], p38 [sc-728 (rabbit), 1:100 and sc-7975 (rabbit) 1:100 respectively] and JNK [sc46009 (goat), 1:100 and sc-6250 (mouse) 1:100 respectively]. After washing with T-PBS, membranes were incubated with donkey antigoat horseradish peroxidase (HRP)-conjugated IgG (sc-2020, 1:1000), bovine anti-rabbit HRP-conjugated IgG (sc-2370, 1:2000), or goat anti-mouse HRP-conjugated IgG (sc-2005, 1:2000) for $60 \mathrm{~min}$ at room temperature in the dark on a shaker. Finally, after three washings with T-PBS, bands were visualized by luminol chemiluminescence (BioRad, Milan, Italy). $\beta$-actin level used as a protein loading control in Western blot.

\section{Densitometric analysis}

The bands obtained after immunoblotting were submitted to densitometric analysis using ID IMAGE ANALYSIS software (Kodak Digital Science). Results were expressed as relative optical density.

\section{Statistical analysis}

The data are presented as means \pm SD. One-way analysis of variance (ANOVA), followed by Tukey's multiple comparison test, was used to evaluate the differences among experimental data. A $P$ value of $<0.05$ was considered significant.

\section{Results}

\section{Molecular markers of apoptosis}

After $16 \mathrm{~h}$ of infection, cells exposed to G. intestinalis trophozoites exhibited the morphological features of apoptosis with characteristic membrane blebbing, chromatin condensation and pyknotic nuclei (data not shown). After staining with YO-PRO-1 dye and PI, apoptotic cells exhibited green fluorescence, dead cells exhibited red and green fluorescence and live cells showed little or no fluorescence (Figure 1a). The percentage of apoptotic cells was significantly $(P<0.001)$ higher after infection with $G$. intestinalis compared to uninfected cells (Figure $1 b)$.

Activation of caspase-3 and PARP fragments, determined by Western blotting, was reported in our previous study, in which we observed that caspase- 3 activation as well as PARP cleavage are activated in HCT-8 cells 16 h post-infection [11]. 


\section{Involvement of MAPKs in G. intestinalis-induced apoptosis}

We also investigated the expression of MAPKs in G. intestinalisinfected HCT-8 cells. We measured only slight phosphorylation of ERK $1 / 2$ after $G$. intestinalis infection (Figure 2). The activation of ERK1/2 was significant $(P<0.05)$ after 20 min of incubation (Figure 2$)$ but the phosphorylated form of ERK was no detectable after shorter (5 min and $10 \mathrm{~min}$ ) or after longer incubation times (30 min to $24 \mathrm{~h}$ ) (data not shown), suggesting early, but transient, activation. In addition, pretreatment with MEK inhibitor PD98059 almost completely inhibited $G$. intestinalis-induced activation. The phosphorylated form of ERK was detectable after $60 \mathrm{~min}$ of actinomycin incubation (data not shown), which is in agreement with observations in other studies [19].

High levels of the phosphorylated forms of p38 and JNK were observed after exposure to the viable trophozoites. Maximum levels of p38 and JNK activation were achieved after $60 \mathrm{~min}$ of incubation. Activation of p38 in response to G. intestinalis infection is shown in (Figure 3), it was clearly activated in infected HCT-8 cells but was barely detectable in control cells. On the contrary, the phosphorylation levels of p38 in G. intestinalis infected cells were not significantly different from those detectable in the controls after both shorter times of incubation ( $5 \mathrm{~min}$ and $10 \mathrm{~min}$ ) and prolonged infection times (more than $60 \mathrm{~min}$ ) (data not shown). Pre-treatment with SB203580 significantly inhibited $G$. intestinalis-induced phosphorylation of p38 MAPK.

JNK is a protein family of serine/threonine kinases encoded by three genes: JNK1, JNK2 and JNK3. The gene products can be alternatively spliced to yield alpha and beta protein forms. Thus, JNK1 and JNK2 can exist either as the full-length $54-\mathrm{kDa}$ protein (p54) or a COOH-terminally truncated $46-\mathrm{kDa}$ form (p46). JNK1 and JNK2 are ubiquitously expressed, including in enterocytes, whereas JNK3 is present in the nervous system and cardiomyocytes but not expressed in the small intestine [18]. We determined the levels of immunoreactive phospho-JNK (p-JNK) in lysates of intestinal cells using Western blot and found the activated JNK form (p46) after $60 \mathrm{~min}$ of infection

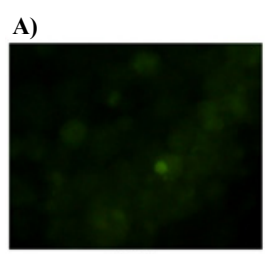

B)

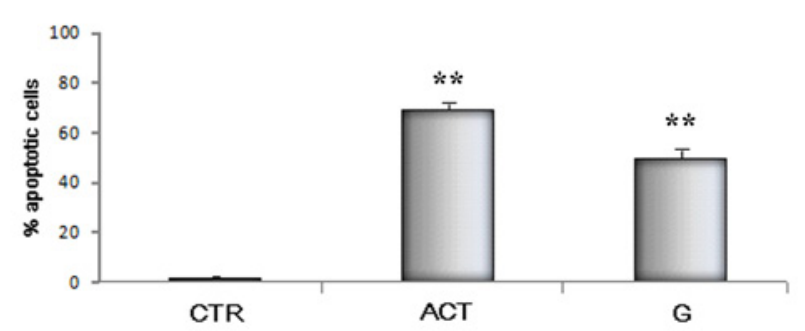

Figure 1: Activation of apoptosis in HCT-8 cells by G. intestinalis. Cells were submitted to different treatments and incubated for $16 \mathrm{~h}$ to evaluate the number of apoptotic cells and caspase- 3 activation. a YO-PRO/PI fluorescent staining. Green, apoptotic cells; red, dead cells. Live cells are unstained. b The percentage of apoptotic cells. CTR, control cells; ACT, actinomycin D-treated cells; G, G. intestinalis-infected cells. Results are expressed as mean \pm SD of five different experiments. ${ }^{* \star} P<0.01$ compared to CTR.

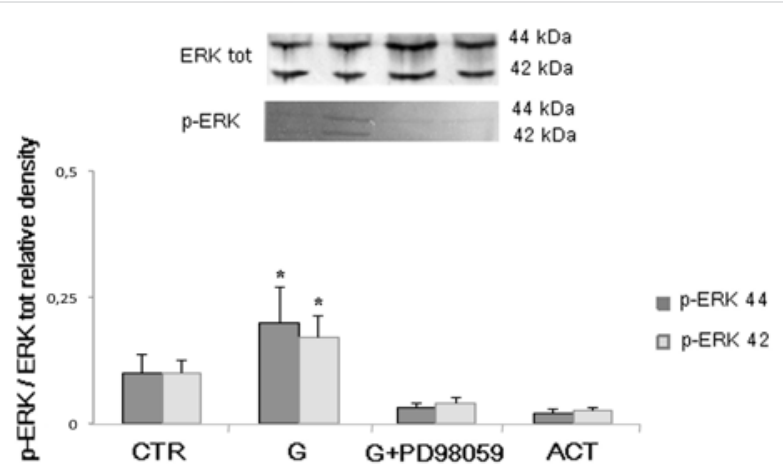

Figure 2: Immunoblotting and densitometric analysis of phosphorylated (p-ERK1/2) and total ERK1/2 protein in HCT-8 cells infected by G. intestinalis after 20 min of incubation. CTR, control cells; G, G. intestinalis-infected cells; G+PD98059, after pre-treatment with the specific inhibitor of ERK1/2 protein; ACT, actinomycin D-treated cells. Results are expressed as mean \pm SD of five different experiments. The protein expression levels were normalized to those of $\beta$-actin and are expressed as means $\pm S D$ of five independent experiments. ${ }^{*} P<0.05$ compared to CTR, ${ }^{* *} P<0.01$ compared to CTR.

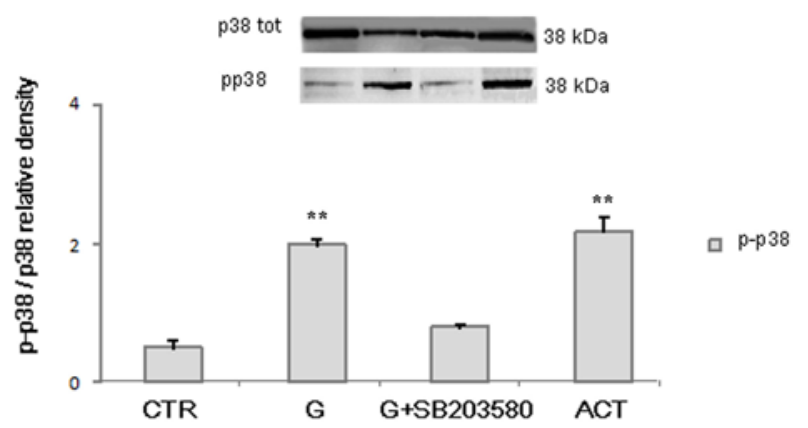

Figure 3: Immunoblotting and densitometric analysis of phosphorylated $(\mathrm{p}$ p38) and total unphosphorylated p38 MAPK protein in HCT-8 cells infected by G. intestinalis after 60 min of incubation. CTR, control cells, G, G. intestinalisinfected cells; G+SB203580, after pre-treatment with the specific inhibitor of p38 protein; ACT, actinomycin D-treated cells. Results are expressed as mean \pm SD of five different experiments. The protein expression levels were normalized to those of $\beta$-actin and are expressed as means \pm SD of five independent experiments. ${ }^{*} \mathrm{P}<0.05$ compared to CTR, ${ }^{* *} \mathrm{P}<0.01$ compared to CTR.

with G. intestinalis (Figure 4). As well as for the p38, the levels of phosphorylated form of JNK in G. intestinalis infected cells were not significantly different from those detectable in the controls after both shorter times of incubation ( $5 \mathrm{~min}$ and $10 \mathrm{~min}$ ) and prolonged infection times (more than $60 \mathrm{~min}$ ) (data not shown). Densitometric analysis revealed that the $\mathrm{p} 46$ form of $\mathrm{p}$-JNK was significantly increased at $60 \mathrm{~min}(P<0.001)$. In addition, pre-treatment with the specific inhibitor SP600125 almost completely inhibited G. intestinalis-induced activation.

Low levels of the phosphorylated forms of MAPKs (ERK1/2, p38 and JNK) were observed in uninfected HCT-8 cells (Figures 2-4). In order to investigate whether the activation of MAPKs is associated with HCT-8 apoptosis induced by $16 \mathrm{~h} \mathrm{G}$. intestinalis infection, we evaluated the inhibitory effect of specific MAPK inhibitors PD98059, SP600125 and SB203580 (which inhibit ERK1/2, JNK and p38, respectively) on the percentage of apoptotic cells. As shown in (Figure 
5), the ERK1/2 inhibitor, PD98059, did not exhibit any inhibitory effect on G. intestinalis-induced apoptosis, whereas SP600125 and SB203580 significantly reduced HCT-8 apoptosis compared to control. Interestingly, when SP600125 and SB203580 were simultaneously added to cell cultures, apoptosis was almost completely suppressed, suggesting the involvement of both p38 MAPK and JNK in the apoptotic process (Figure 5).

Finally, we examined the role of MAPKs in caspase- 3 activation following $16 \mathrm{~h}$ incubation with $G$. intestinalis. We observed that pretreatment with the different inhibitors significantly reduced $(P<0.001)$ caspase- 3 expression in HTC-8 cells. Though the ERK1/2 inhibitor weakly reduced the levels of caspase-3, both SP600125 and SB203580 were able to strongly reduce the expression of the cleaved form of caspase-3 and to block this expression when simultaneously added

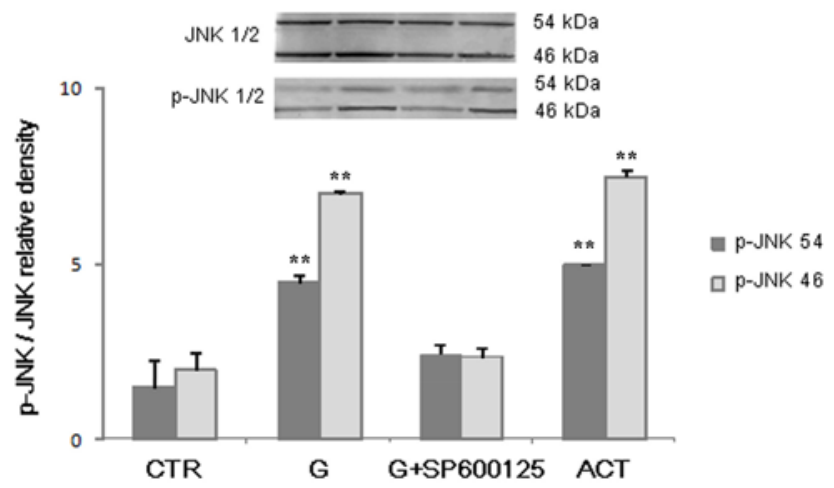

Figure 4: Immunoblotting and densitometric analysis of phosphorylated (pJNK1/2) and total unphosphorylated (JNK1/2) protein in HCT-8 cells infected by $G$. intestinalis after 60 min of incubation. CTR, control cells; G, G. intestinalis-infected cells; G+SP600125, after pre-treatment with the specific inhibitor of JNK1/2; ACT, actinomycin D-treated cells. Results are expressed as mean \pm SD of five different experiments. The protein expression levels were normalized to those of $\beta$-actin and are expressed as means \pm SD of five independent experiments. ${ }^{*} P<0.05$ compared to CTR, ${ }^{* *} P<0.01$ compared to CTR.

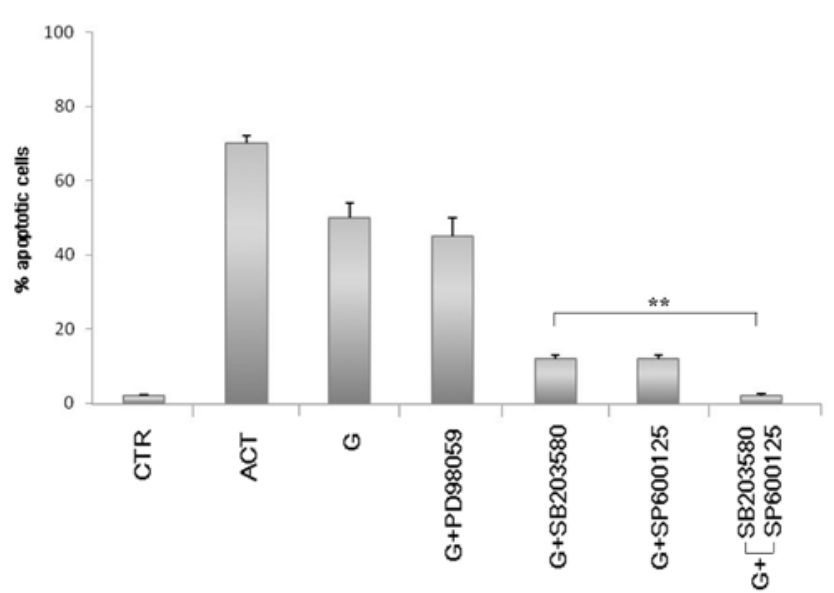

Figure 5: Involvement of MAPKs in HCT-8 cell apoptosis induced by G. intestinalis after $16 \mathrm{~h}$ of incubation. Percentage of apoptosis in unstimulated cells (CTR), actinomycin D-treated cells (ACT), G. intestinalis-infected cells $(\mathrm{G})$, and G. intestinalis-infected cells after pre-treatment with PD98059, SB203580, SP600125, or SB203580 + SP600125. Results are expressed as means \pm SD of five independent experiments. ${ }^{* *} P<0.01$ compared to $\mathrm{G}$.

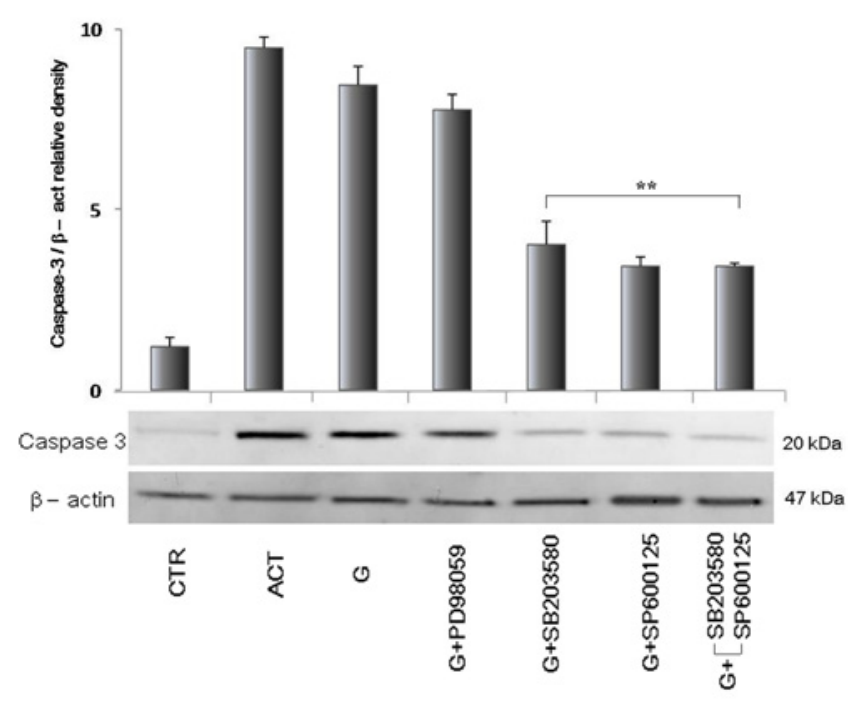

Figure 6: Involvement of MAPKs in caspase-3 activation by $G$. intestinalis after $16 \mathrm{~h}$ of incubation. Immunoblotting and densitometric analysis of caspase-3 activation in unstimulated cells (CTR), actinomycin D-treated cells (ACT), G. intestinalis-infected cells $(G)$, and $G$. intestinalis-infected cells after pretreatment with PD98059, SB203580, SP600125, or SB203580 + SP600125. Results are expressed as means \pm SD of five independent experiments. ${ }^{* *} P$ $<0.01$ compared to $\mathrm{G}$.

to cells (Figure 6). These results suggest that p38 MAPK and JNK are involved in the activation of intracellular caspase-3 in G. intestinalisinfected cells.

\section{Discussion}

Apoptosis can be induced by a variety of different signals, including activation of Fas or tumor necrosis factor receptors, deprivation of growth factors and different stresses and is mediated by the caspase enzymes. Several pathogens have been reported to trigger apoptosis or modulate apoptosis by interfering with the cell survival pathways. Here, we report that $G$. intestinalis induces apoptosis through the activation of caspase- 3 in the human intestinal cell line HCT-8. The present study demonstrates that MAPK activation plays an important role in the regulation of apoptosis induced by $G$. intestinalis. Incubation of HCT8 cells with viable trophozoites of $G$. intestinalis resulted in a significant increase in MAPK activation. In particular, ERK was barely activated in the early phase of Giardia infection, whereas a strong activation of both JNK and p38 MAPK was detected in Giardia-infected cells after longer incubation periods. MAPK activation seems to correlate with regulation of the apoptotic process, as specific MAPK inhibitors significantly reduced the expression of the active form of caspase-3 in infected cells. Finally, we demonstrated that apoptotic changes were dramatically inhibited by pre-treating the cells with both the JNK and p38 specific inhibitors, but not the ERK1/2 inhibitor. Taken together, these results suggest a critical role for MAPK activation in $G$. intestinalis-induced apoptosis in HCT-8 cells.

Apoptosis is well known to be under tight regulatory control. Among these regulators, MAPKs are important mediators of apoptosis in all eukaryotic organisms [19]. In mammalian cells, at least three MAPKs subtypes are known, namely ERK1/2, p38 and JNK. The ERK cascade is mainly activated through receptor-mediated signaling stimuli, including growth factors and is associated with cell proliferation, differentiation and contributes to some cases of cell death 
[20,21]. In contrast, p38 and JNK (also called stress-activated protein kinase, SAPK) cascades are activated in response to treatment with proinflammatory or stressful stimuli, including bacterial and viral infection and appear to be closely related to cell death [22-25]. Apoptosis is an important factor in both parasite survival and pathogenicity [26-28], exhibiting multifaceted roles in host-pathogen interactions. In regards to Giardia infection, previous studies have indicated a relationship between enterocyte apoptosis and loss of the epithelial barrier function in a human duodenal epithelial cell line infected with trophozoites of certain strains of G. lamblia [29], though the mechanisms underlying this process are still under investigation.

Several reports in the literature suggest a pivotal role for MAPKs in the regulation of apoptosis by intracellular parasites. For example, the p38 MAPK signaling cascade is a requisite for the apoptosis of Trichomonas vaginalis-infected macrophages, via the phosphorylation of p38 MAPK [30]. Yersinia enterocolitica down-regulates JNK, p38 MAPK and ERK1/2 in macrophages. Moreover, evidence suggests that Entamoeba histolytica induces apoptosis of host cells, including neutrophils, $\mathrm{T}$ lymphocytes and macrophages and that ERK1/2 activation is required for this process [31].

Other evidence has shown that infection with the promastigote forms of Leishmania major and Leishmania donovani induces infected cells to resist apoptosis [27,32-34] and that survival of the intracellular protozoan correlates with attenuation of MAPK signaling, though the role for kinases in the apoptotic program is controversial. In fact, several investigators have reported that signaling through p38 MAPK, like other signaling pathways, is either avoided by Leishmania parasites or actively suppressed [35]. Nonetheless, at least two reports have demonstrated the appearance of phosphorylated p38 MAPK soon after infection with Leishmania [36,37]. Finally, p38 MAPK and JNK signaling cascades have also been shown to be involved in apoptosis induced by Reoviridae family members, including HIV-1, herpes simplex virus 1 and hepatitis $C$ virus. The molecular basis for these different observations is still unknown.

Therefore, the involvement of ERK1/2, JNK and p38 MAPK during apoptosis regulation is dependent upon different kinds of stimuli and cell types. The MAPK-mediated signaling pathways demonstrate that activation of JNK and p38 MAPK with concurrent inhibition of ERK is critical to the induction of apoptosis [25]. Therefore, the dynamic balance between growth factor-activated ERK and stress-activated JNK-p38 pathways may be important in determining whether a cell survives or undergoes apoptosis.

The induction of host cell death observed during microbial (bacterial, viral, parasitic) infection has important consequences on pathogenesis. The death of an infected cell may represent a defense mechanism for promoting efficient pathogen elimination by hampering its replication and avoiding dissemination of the microorganism in the host. However, if excessive, apoptosis is pathogenic, as observed during sepsis in which lymphocyte apoptosis is responsible for immunosuppression [38]. In regards to giardiasis, one can postulate that the Giardia-induced apoptotic process, if persistent or uncontrolled, may be pathogenic because it is responsible for epithelial barrier loss, explaining the dysregulation of bowel permeability observed during Giardia infection.

\section{Conclusions}

In summary, the results of the present study provide the first evidence that live trophozoites of $G$. intestinalis are able to induce apoptosis in HCT-8 cells through the activation of MAPKs JNK and

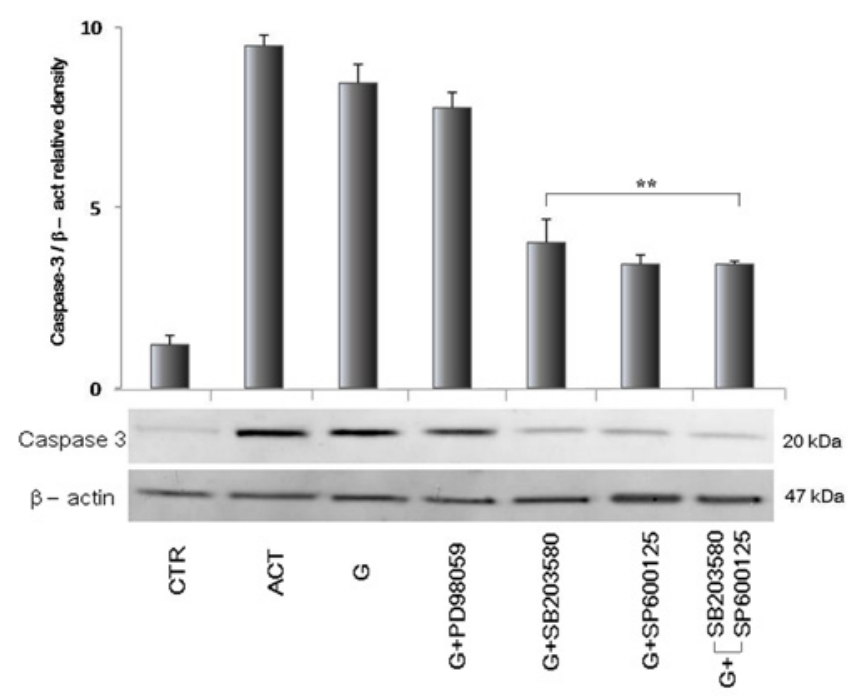

Figure 7: Schematic representation of MAPK-dependent apoptosis in G. intestinalis-infected HCT-8 cells. Phosphorylation of both p38 MAPK and JNK increased after exposure to the viable trophozoites, leading to cell apoptosis through caspase-3 activation (right). Specific inhibition of p38 MAPK and JNK impaired caspase-3 activation, ensuring cell survival (left). SP600125 and SB203580 are specific inhibitors of JNK and p38 phosphorylation, respectively.

p38 (Figure 7). The present study offers important insights that may help us gain a better understanding of the molecular mechanisms involved in Giardia-associated pathogenesis.

\section{Acknowledgements}

We have no competing financial interests

This study was supported in part by a "Dottorato di Ricerca" grant entitled "Morfobiologia applicata e citometabolismo dei farmaci".

We thank Mary Victoria Pragnell, B.A., for English language assistance.

\section{References}

1. Thompson RC (2000) Giardiasis as a re-emerging infectious disease and its zoonotic potential. Int J Parasitol 30: 1259-1267.

2. Hellard ME, Sinclair MI, Hogg GG, Fairley CK (2000) Prevalence of enteric pathogens among community based asymptomatic individuals. J Gastroentero Hepatol 15: 290-293.

3. Rodriguez-Hernandez J, Canut-Blasco A, Martin-Sanchez AM (1996) Seasonal prevalences of Cryptosporidium and Giardia infections in children attending day care centres in Salamanca (Spain) studied for a period of 15 months. Eur J Epidemiol 12: 291-295.

4. Buret AG (2008) Pathophysiology of enteric infections with Giardia duodenalius Parasite 15: 261-265.

5. D'Anchino M, Orlando D, De Feudis L (2002) Giardia lamblia infections become clinically evident by eliciting symptoms of irritable bowel syndrome. J Infect 45 169-172.

6. Di Prisco MC, Hagel I, Lynch NR, Barrios RM, Alvarez N, et al. (1993) Possible relationship between allergic disease and infection by Giardia lamblia. Ann Allergy 70: 210-213.

7. Carlson DW, Finger DR (2004) Beaver fever arthritis. J Clin Rheumatol 10 86-88.

8. Thompson RC (2004) The zoonotic significance and molecular epidemiology of Giardia and giardiasis. Vet Parasitol 126: 15-35.

9. Xiao L, Fayer R (2008) Molecular characterisation of species and genotypes of Cryptosporidium and Giardia and assessment of zoonotic transmission. Int $J$ Parasitol 38: 1239-1255 
Citation: Cavallo P, Cianciulli A, Calvello R, Dragone T, Panaro MA (2012) MAP Kinases are Required for Apoptosis of HCT-8 Epithelial Cells Induced by the Parasite Giardia intestinalis. J Microbial Biochem Technol 4: 031-036. doi:10.4172/1948-5948.1000068

10. Muller N, von Allmen N (2005) Recent insights into the mucosal reactions associated with Giardia lamblia infections. Int J Parasitol 35: 1339-1347.

11. Panaro MA, Cianciulli A, Mitolo V, Mitolo Cl, Acquafredda A, et al. (2007) Caspase-dependent apoptosis of the HCT-8 epithelial cell line induced by the parasite Giardia intestinalis. FEMS Immunol Med Microbiol 51: 302-309.

12. Junttila MR, Li SP, Westermarck J (2008) Phosphatase-mediated crosstalk between MAPKs signaling pathways in the regulation of cell survival. FASEB J 22: 954-965

13. Kostenko S, Dumitriu G, Lægreid KJ, Moens U (2011) Physiological roles of mitogen-activated-protein-kinase-activated p38-regulated/activated protein kinase. World J Biol Chem 2: 73-89

14. Pearson G, Robinson F, Beers Gibson T, Xu BE, Karandikar M, et al. (2001) Mitogen-activated protein (MAP) kinase pathways: regulation and physiological functions. Endocr Rev 22: 153-183.

15. Keister DB (1983) Axenic culture of Giardia lamblia in TYI-S-33 medium supplemented with bile. Trans R Soc Trop Med Hyg 77: 487-488.

16. Bradford MM (1976) A rapid and sensitive method for the quantitation of microgram quantities of protein utilizing the principle of protein-dye binding. Anal Biochem 72: 248-254.

17. Yamada M, Banno Y, Takuwa Y, Koda M, Hara A (2004) Overexpression of phospholipase D prevents actinomycin D-induced apoptosis through potentiation of phosphoinositide 3-kinase signalling pathways in Chinesehamster ovary cells. Biochem J 378: 649-656.

18. Davis RJ (2000) Signal transduction by the JNK group of MAP kinases. Cell 103: $239-252$.

19. English J, Pearson G, Wilsbacher J, Swantek J, Karandikar M, et al. (1999) New insights into the control of MAP kinase pathways. Exp Cell Res 253: 255270 .

20. Stanciu M, Wang Y, Kentor R, Burke N, Watkins S, et al. (2000) Persistent activation of ERK contributes to glutamate-induced oxidative toxicity in a neuronal cell line and primary cortical neuron cultures. J Biol Chem 275: $12200-$ 12226

21. Wang X, Martindale JL, Holbrook NJ (2000) Requirement for ERK activation in cisplatin-induced apoptosis. J Biol Chem 275: 39435-39443.

22. Chen YR, Wang X, Templeton D, Davis RJ, Tan TH (1996) The role of c-Jun $\mathrm{N}$-terminal kinase (JNK) in apoptosis induced by ultraviolet $\mathrm{C}$ and gamma radiation. Duration of JNK activation may determine cell death and proliferation. J Biol Chem 13: 31929-33196.

23. Graves JD, Draves KE, Craxton A, Saklatvala J, Krebs EG (1996) Involvement of stress-activated protein kinase and p38 mitogen-activated protein kinase in mlgM-induced apoptosis of human B lymphocytes. Proc Natl Acad Sci 93: 13814-13818.

24. Holloway G, Coulson BS (2006) Rotavirus activates JNK and p38 signaling pathways in intestinal cells, leading to AP-1-driven transcriptional responses and enhanced virus replication. J Virol 80: 10624-10633.

25. Xia Z, Dickens M, Raingeaud J, Davis RJ, Greenberg ME (1995) Opposing effects of ERK and JNK-p38 MAP kinases on apoptosis. Science 270: 13261331.

26. James ER, Green DR (2004) Manipulation of apoptosis in the host-parasite interaction. Trends Parasitol 20: 280-287.

27. Lisi S, Sisto M, Acquafredda A, Spinelli R, Schiavone M, et al. (2005) Infection with Leishmania infantum Inhibits actinomycin D-induced apoptosis of human monocytic cell line U-937. J Eukaryot Microbiol 52: 211-217.

28. Picot S (2006) Apoptosis and programmed cell death. Host parasite relationship new paradigm. Med Trop (Mars) 66: 111-117.

29. Chin AC, Teoh DA, Scott KG, Meddings JB, Macnaughton WK, et al. (2002) Strain-dependent induction of enterocyte apoptosis by Giardia lamblia disrupts epithelial barrier function in a caspase-3-dependent manner. Infect Immun 70: 3673-3680.

30. Chang JH, Kim SK, Choi IH, Lee SK, Morio T, et al. (2006) Apoptosis of macrophages induced by Trichomonas vaginalis through the phosphorylation of p38 mitogen-activated protein kinase that locates at downstream of mitochondria-dependent caspase activation. Int J Biochem Cell Biol 38: 638647.

31. Sim S, Yong TS, Park SJ, Im KI, Kong Y, et al. (2005) NADPH oxidasederived reactive oxygen species-mediated activation of ERK $1 / 2$ is required for apoptosis of human neutrophils induced by Entamoeba histolytica. J Immunol 174: 4279-4288.

32. Moore KJ, Matlashewski G (1994) Intracellular infection by Leishmania donovani inhibits macrophage apoptosis. J Immunol 152: 2930-2937.

33. Aga E, Katschinski DM, van Zandbergen G, Laufs H, Hansen B, et al. (2002) Inhibition of the spontaneous apoptosis of neutrophil granulocytes by the intracellular parasite Leishmania major. J Immunol 169: 898-905.

34. Akarid K, Arnoult D, Micic-Polianski J, Sif J, Estaquier J, et al. (2004) Leishmania major-mediated prevention of programmed cell death induction in infected macrophages is associated with the repression of mitochondrial release of cytochrome c. J Leukoc Biol 76: 95-103.

35. Olivier M, Gregory DJ, Forget G (2005) Subversion mechanisms by which Leishmania parasites can escape the host immune response: a signaling poin of view. Clin Microbiol Rev 18: 293-305.

36. Junghae M, Raynes JG (2002) Activation of p38 mitogen-activated protein kinase attenuates Leishmania donovani infection in macrophages. Infect Immun 70: 5026-5035

37. Balaraman S, Singh VK, Tewary P, Madhubala R (2005) Leishmania lipophosphoglycan activates the transcription factor activating protein 1 in J774A.1 macrophages through the extracellular signal-related kinase (ERK) and p38 mitogen-activated protein kinase. Mol Biochem Parasitol 139: 117 127.

38. Hotchkiss RS, Nicholson DW (2006) Apoptosis and caspases regulate death and inflammation in sepsis. Nat Rev Immunol 6: 813-822. 